\title{
Alternative splicing coupled with transcript degradation modulates OAS1g antiviral activity
}

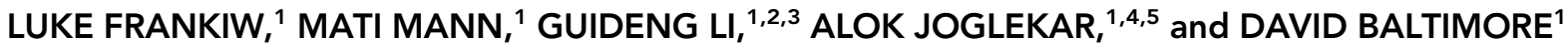 \\ ${ }^{1}$ Division of Biology and Biological Engineering, California Institute of Technology, Pasadena, California 91125, USA \\ ${ }^{2}$ Center of Systems Medicine, Institute of Basic Medical Sciences, Chinese Academy of Medical Sciences and Peking Union Medical College, \\ Beijing 100005, China \\ ${ }^{3}$ Suzhou Institute of Systems Medicine, Suzhou 215123, China \\ ${ }^{4}$ Center for Systems Immunology, University of Pittsburgh, Pittsburgh, Pennsylvania 15261, USA \\ ${ }^{5}$ Department of Immunology, University of Pittsburgh, Pittsburgh, Pennsylvania 15261, USA
}

\begin{abstract}
At the heart of an innate immune response lies a tightly regulated gene expression program. This precise regulation is crucial because small changes can shift the balance from protective to destructive immunity. Here we identify a frequently used alternative splice site in the gene oligoadenylate synthetase $1 \mathrm{~g}$ (Oas $1 \mathrm{~g}$ ), a key component of the 2-5A antiviral system. Usage of this splice site leads to the generation of a transcript subject to decay, and removal of the site leads to increased expression of Oas1g and an improved antiviral response. However, removal of the splice site also leads to an increase in apoptotic cell death, suggesting this splicing event exists as a compromise between the pathogen protective benefits and collateral damage associated with OAS1g activity. Across the innate immune response, we show that a multitude of alternative splicing events predicted to lead to decay exist, and thus have the potential to play a significant role in the regulation of gene expression in innate immunity.
\end{abstract}

Keywords: AS-NMD; alternative splicing; antiviral response; oligoadenylate synthetase; posttranscriptional regulation

\section{INTRODUCTION}

Central to an inflammatory response is a robust and coordinated gene expression program. Precise regulation of this gene expression program is crucial to avoid immunemediated collateral damage (Kontoyiannis et al. 1999). Though transcription and protein turnover are the best-examined areas of gene expression regulation (Gautier et al. 2012; Chen and Chen 2013; Smale and Natoli 2014; Smale et al. 2014), a variety of posttranscriptional mechanisms have emerged that play a role in the fine-tuning of an inflammatory response. Well-studied examples include mRNA stabilization (Hao and Baltimore 2009), mRNA deadenylation (Leppek et al. 2013), and microRNA regulation (O'Connell et al. 2012).

The wealth of transcriptomic data generated over the last decade has shed light on the widespread nature of alternative mRNA splicing of mammalian genes. While most mammalian genes exhibit alternative splicing (AS) (Pan et al. 2008; Wang et al. 2008), not all of the produced transcripts encode functional proteins. Though AS can act to

Corresponding author: baltimo@caltech.edu

Article is online at http://www.rnajournal.org/cgi/doi/10.1261/rna. 073825.119. increase proteomic diversity, it can also generate unproductive isoforms subject to either cytoplasmic or nuclear decay (Lareau et al. 2007a; Bitton et al. 2015). In cases where a splicing event leads to the introduction of a premature termination codon (PTC), degradation is believed to occur via the nonsense-mediated mRNA decay (NMD) machinery in the cytoplasm through a process called alternative splicing-coupled NMD (AS-NMD) (Lareau et al. 2007a; Kervestin and Jacobson 2012; Jangi and Sharp 2014). Coupling of AS to NMD provides cells with a potential mode of down-regulation of expression of a given gene. It has been estimated that 10\%-30\% of mammalian genes may be regulated posttranscriptionally through ASNMD (Lewis et al. 2003; Mendell et al. 2004; Weischenfeldt et al. 2012; Jangi and Sharp 2014). However, beyond splicing factor regulation (Lareau et al. 2007b; Jangi and Sharp 2014), the extent to which AS-NMD represents posttranscriptional gene expression control as opposed to noise in the splicing process is a contentious issue, and

(C) 2020 Frankiw et al. This article is distributed exclusively by the RNA Society for the first 12 months after the full-issue publication date (see http://rnajournal.cshlp.org/site/misc/terms.xhtml). After 12 months, it is available under a Creative Commons License (Attribution-NonCommercial 4.0 International), as described at http:// creativecommons.org/licenses/by-nc/4.0/. 
the role it plays in the finely tuned innate immune response has yet to be explored (McGlincy and Smith 2008).

Here we identify a frequently used unproductive splicing event in oligoadenylate synthetase $1 \mathrm{~g}$ (Oas1g), an important murine antiviral response factor. Upon binding viral dsRNA, OAS1g acts to convert ATP into $2^{\prime}-5^{\prime}$ linked oligoadenylates (2-5A), which in turn activate RNase L. Activated RNase L degrades viral RNA, in turn inhibiting viral replication and propagation (Silverman 2007). Although humans have a single Oas1 gene, in mice the Oas1 gene locus underwent a series of duplication events leading to the existence of eight Oas1 paralogs. Of the paralogs, only OAS1a and OAS1g have been shown to be enzymatically active, with OAS1g producing significantly more oligoadenylates as compared to OAS1a (Kakuta et al. 2002; Elkhateeb et al. 2016). We show that removal of the Oas1g alternative splice site in a murine macrophage cell line leads to increased expression of Oas1g, both in stimulated and unstimulated conditions. Further, this increased expression of Oas $1 \mathrm{~g}$ improves the ability of macrophages that lack the unproductive splice site to withstand infection by encephalomyocarditis virus (EMCV). However, removal of the Oas1g alternative splice site also leads to an increase in apoptotic cell death in uninfected cells, a finding consistent with the idea that activation of the 2-5A antiviral system can be detrimental to host fitness (Zhou et al. 1997; Andersen et al. 2007; Carey et al. 2019). Beyond Oas1g, we find AS-NMD events in a number of other crucial innate immune response transcripts, suggesting this is a common mechanism of mitigation for responses that might otherwise be unchecked or inappropriately scaled.

\section{RESULTS}

\section{Oas1g has a frequent AS-NMD event}

AS events have the potential to generate both productive isoforms coding for functional proteins, as well as unproductive isoforms subject to degradation (Fig. 1A). The latter allows for the use of AS as a posttranscriptional mechanism of gene-expression regulation. To investigate the extent to which unproductive splicing acts as a posttranscriptional regulator of gene expression during inflammation, we analyzed nuclear fractionation RNA-sequencing data from mouse bone marrow-derived macrophages (BMDMs) stimulated with the TLR3 agonist poly(l:C) for up to $12 \mathrm{~h}$ (Frankiw et al. 2019). Activation of TLR3 leads to activation of interferon regulator factors, production of interferon- $\alpha$ and $\beta(\mathrm{IFN}-\alpha / \beta)$, and induction of a type I interferon response (Perales-Linares and Navas-Martin 2013). RNAseq samples were derived from total nuclear RNA so as to avoid underrepresentation of isoforms subjected to NMD decay, which occurs rapidly in the cytoplasm. From this data, we identified frequent usage of an alternative $5^{\prime}$ splice site at the third splice junction in the Oas1g gene (Fig. 1B). At each time-point we analyzed, this alternative "unproductive" splice site was frequently selected over the consensus "productive" splice site (Fig. 1C, left). This is evident by comparing the number of reads that map across the two different junctions, as well as through the use of the computational program MISO, which utilizes a probabilistic framework to estimate the expression of alternatively spliced isoforms (Fig. 1C, right; Katz et al. 2010). The metric percent spliced in (PSI; $\Psi$ ) is an estimate of the fraction of transcripts that utilize the alternative unproductive splice site, and the associated histograms represent the posterior distributions over $\Psi$. For each individual time-point, derived from a single RNA-seq sample, the mean $\Psi$ is depicted by the red line on the histogram, and its value is labeled along with the $95 \%$ confidence intervals of the distribution. Higher $\Psi$ values indicate increased usage of the unproductive splice isoform. The unproductive splice isoform of Oas $1 \mathrm{~g}$ represents an ideal NMD substrate as it contains a termination codon located $>50$ nt upstream of a downstream exon-exon junction. As might be expected, depletion of the NMD factor Upf1 leads to increased expression levels of Oas $1 \mathrm{~g}$ (Supplemental Fig. S1A,B). Of interest was the strength of the productive and unproductive splice site, which can be quantified using a maximum entropy model (Yeo and Burge 2004). We find the productive and unproductive $5^{\prime}$ splice sites are similar in strength, and are fairly strong with respect to all expressed junctions (Supplemental Fig. S1C).

Next, we looked at this AS event in the context of all expressed junctions. To do this, we calculated the alternative junction usage at each expressed junction from the BMDM data-set stimulated with poly(l:C) for $8 \mathrm{~h}$ (Fig. 1D, data from other induced time-points shown in Supplemental Fig. S1D,E). From this junction-centric viewpoint, the sequencing data supports the conclusion that most expressed junctions splice with high fidelity (Fig. 1E). Still, there is some alternative junction usage, which can be attributed to both regulated AS events as well as splicing noise. With respect to the alternatively spliced junction of Oas $1 \mathrm{~g}$, it ranks near the top percentile of alternative junction usage, supporting the conclusion that this AS event is among the most frequently utilized in poly(I:C) stimulated BMDMs (Fig. 1E).

\section{Removal of alternative splice site alters Oas1g expression and host response to EMCV}

In order to explore the effect of this AS event on Oas1g expression, and correspondingly the antiviral response, we used clustered regularly interspaced short palindromic repeats (CRISPR)-CRISPR-associated protein-9 nuclease (Cas9) technology to engineer murine RAW 264.7 cell lines devoid of this unproductive splice site (Fig. 2A). In parallel, cell lines expressing Cas9 and a nontargeting guide were 


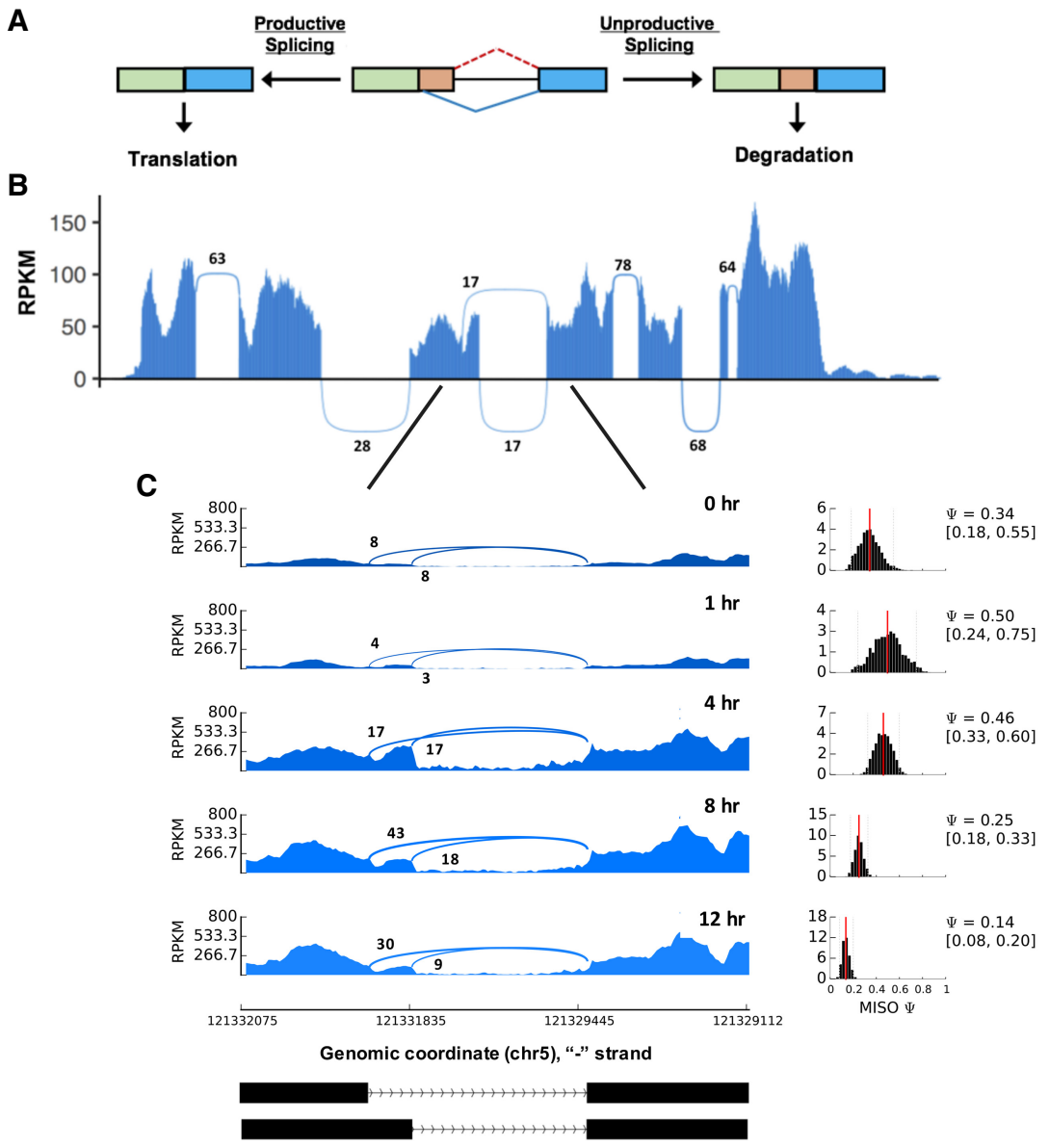

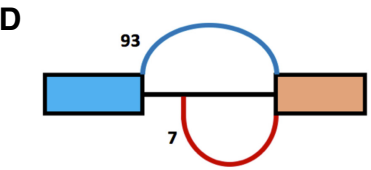

Alt. Junction Usage $=\frac{7}{93+7}=0.07$

$\mathbf{E}$

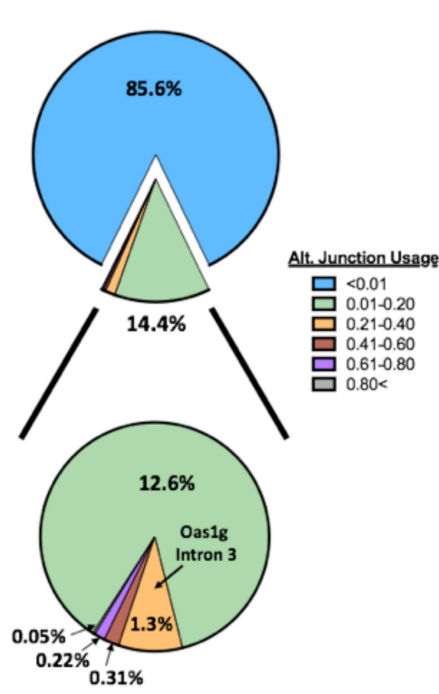

FIGURE 1. Oas1g has a frequent AS-NMD event. (A) A schematic depiction of an AS event leading to either a productive isoform destined for translation or an unproductive isoform destined for degradation. (B) Sashimi plot for the entire gene body of Oas1 $\mathrm{g}$ from BMDMs stimulated with poly $(\mathrm{I}: \mathrm{C})$ for $12 \mathrm{~h}$. Sequenced RNA is derived from the total nuclear fraction. Oas $1 \mathrm{~g}$ is a negative strand gene and is oriented such that the negative strand runs left to right. (C) (Left) Sashimi plots centered at the third junction of Oas1g from BMDMs stimulated with poly(l:C) for 0, 1, 4, 8, and $12 \mathrm{~h}$. Sequenced RNA is derived from the total nuclear fraction. The $y$-axis represents reads per kilobase of transcript, per million mapped reads (RPKM). (Right) Posterior distributions of the $\Psi$ value for each individual time point. The mean $\Psi$ is depicted by the red line. Mean and $95 \%$ confidence intervals are labeled to the right of the posterior distribution. (D) Schematic representation of the alternative junction usage calculation. (E) Pie chart representing alternative junction usage for all expressed junctions upon $8 \mathrm{~h}$. of poly(l:C) stimulation. The slice including the alternatively spliced third junction of Oas1g is labeled (alt. junction usage $0.21-0.40$ ). Genomic coordinates represent the mm9 genome assembly.

generated. We selected seven clones that had the splice site removed in both alleles, which we designate as "fixed" clones (Fig. 2B; Supplemental Fig. S2). RT-PCR with RNA from whole-cell fractions that was stimulated with poly(l: C) both confirmed alternative splice site usage in control populations, and showed forced productive splicing in these fixed clones (Fig. 2C).

To determine what effect this forced productive splicing has on Oas1g expression, we monitored mRNA levels of Oas1g in both unstimulated and stimulated [8 h poly(l:C)] conditions. In each case, the engineered lines lacking the unproductive Oas1g splice site had significantly higher levels of expression, presumably due to lack of NMD associated with selection of the unproductive splice site (Fig. 2D). Of interest, levels of Oas1g in unstimulated Oas1g splice site engineered cells were similar to levels of
Oas1g in stimulated control cells. Next, to determine the effect of removal of the unproductive splice site with respect to the antiviral response, we used EMCV to infect both groups of macrophages. EMCV is a (+)ssRNA member of the Picornaviridae family that replicate through partially dsRNA intermediates (Carocci and Bakkali-Kassimi 2012). Infection has been shown to cause accumulation of 2-5A, and viral replication is sensitive to the OAS/ RNase L pathway (Hearl and Johnston 1987; Zhou et al. 1997). As oligoadenylate synthetases bind viral dsRNA, the RNA activators in EMCV-infected cells are believed to be the viral replicative intermediates (Silverman 2007). Upon $18 \mathrm{~h}$ of infection with EMCV, we again observed significantly higher levels of Oas1g expression in the engineered lines lacking the unproductive Oas1g splice site (Fig. 2E). Using qPCR to measure levels of EMCV following 
A

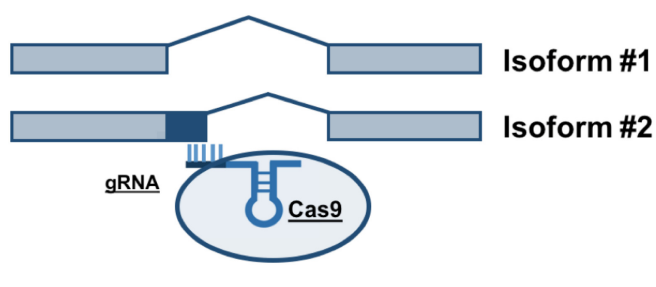

B

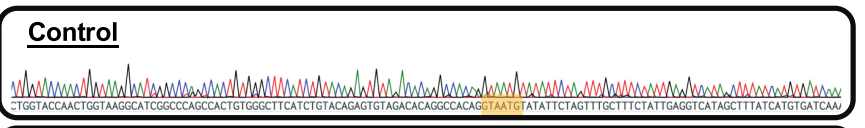

Fixed Clone \#1

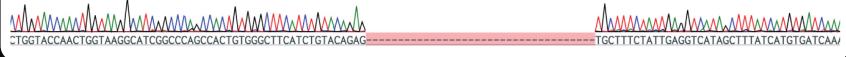

C

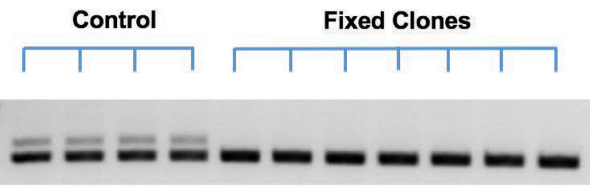

D

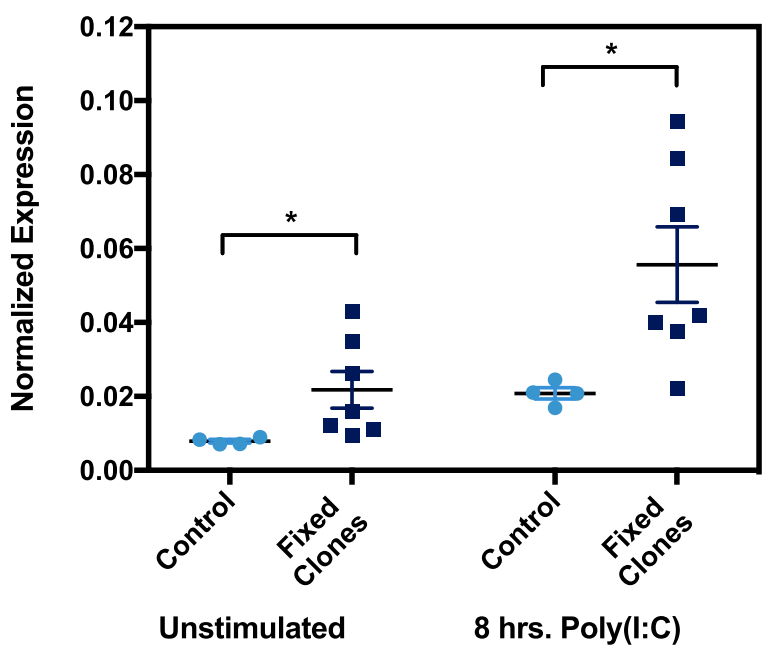

E
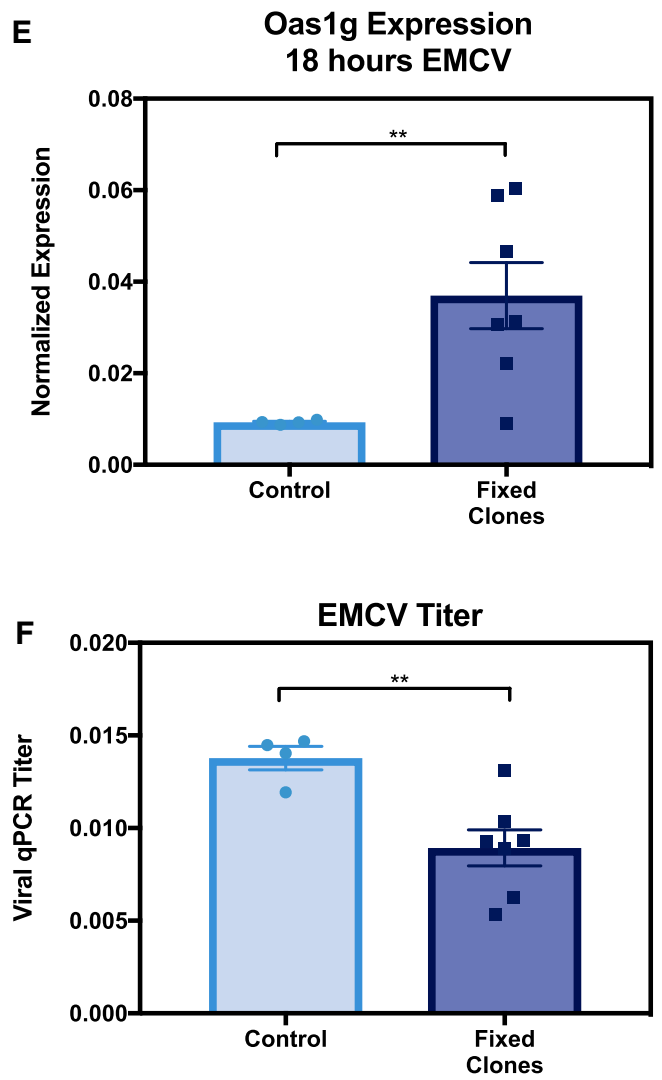

G

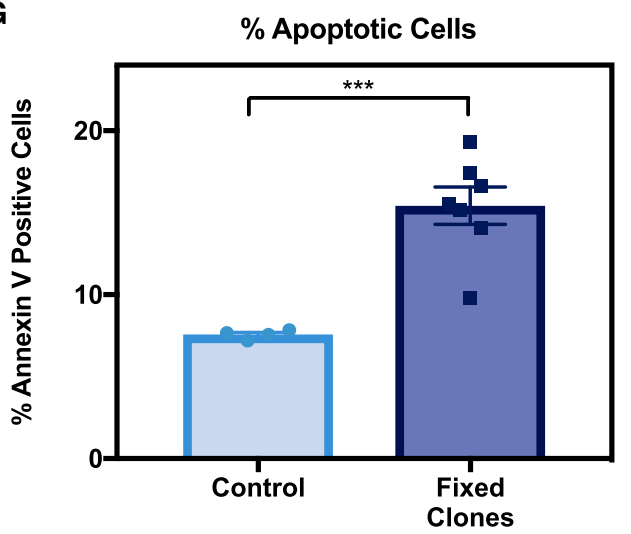

FIGURE 2. Removal of alternative splice site alters Oas1g expression and macrophage response to EMCV. (A) Schematic representation of the two alternative splice isoforms, and the gRNA/Cas9 targeting of the alternative splice site. (B) Sanger sequencing gDNA from a control sample (top) and an Oas1 SS KO sample (bottom). Sequencing is oriented such that the negative strand runs left to right. The alternative splice site is represented by the yellow highlighted region. (C) RT-PCR upon stimulation with poly(l:C) confirming alternative splice site usage in control populations and forced productive splicing in fixed clones. (D) RT-qPCR analysis of Oas1g mRNA levels in unstimulated and stimulated [8 h poly(l:C)] macrophages. Control samples are represented in light blue, SS KO clones are represented in dark blue. (E) RT-qPCR analysis of Oas $1 \mathrm{~g} m R N A$ levels in EMCV infected (18 h) macrophages. Control samples are represented in light blue, SS KO clones are represented in dark blue. (F) RT-qPCR measurement of EMCV viral load following $18 \mathrm{~h}$ of infection at $1 \mathrm{MOI}$. Control samples are represented in light blue, SS KO clones are represented in dark blue. (G) Annexin V staining for apoptotic cells under unstimulated conditions. Control samples are represented in light blue, SS KO clones are represented in dark blue. Data is representative of two independent experiments $(D-G)$ and is shown as mean (error bars indicate $S E M) .(*) P<0.05$, (**) $P<0.01$, and $(* * *) P<0.001$ using a Student's t-test. Results are presented relative to those of Rpl32 (D-F).

$18 \mathrm{~h}$ of infection, we found that the engineered lines controlled viral replication more efficiently than the control lines (Fig. 2F). While this effect was relatively minor (approximately twofold), the effect was consistent across the clones and was statistically significant. Thus, we conclude that forced productive splicing of Oas1g improves the antiviral defense through increased expression of Oas1g. Finally, since activation of the $2-5 A$ system has been 
shown to promote apoptosis in host cells (Castelli et al. 1997), we were interested in determining whether removal of the unproductive Oas1 $\mathrm{g}$ splice site altered the levels of apoptotic cells. We observed approximately twofold increase in the fraction of apoptotic cells in the engineered lines lacking the unproductive Oas $1 \mathrm{~g}$ splice site as compared to the control cells (Fig. 2G) in unstimulated conditions. We conclude that the increased Oas1g observed with the removal of the unproductive splice site leads to increased levels of apoptosis in a cell population.

Of note, the other enzymatically active member of the murine Oas1 family, Oas1a, has a highly homologous junction with an identical unproductive splice site. However, despite nearly complete similarity of sequence at and near this splice-site (Supplemental Fig. S3A), it is used less frequently than that of Oas1g (Supplemental Fig. 3B-D). Because of this similarity, our guide targeted to the unproductive splice site of Oas $1 \mathrm{~g}$ also cut at Oas1a (Supplemental Fig. S4), and genotyping confirmed all selected clones deleted the Oas1a unproductive splice site in addition to the Oas1g unproductive splice site. Again, RT-PCR with RNA whole-cell fractions upon stimulation with poly(l:C) confirmed alternative splice site usage in control populations, and showed forced productive splicing in edited clones (Supplemental Fig. S3C). To determine what effect this forced productive splicing has on Oas1a expression, we monitored Oas1a mRNA levels in both unstimulated and stimulated [ $\mathrm{h}$ poly(l:C)] conditions. In this case, we found that while the mean expression of Oas1a in both unstimulated and stimulated conditions was greater in nonengineered clones, the effect lacked significance (Supplemental Fig. S3D). We hypothesize that the dampened effect with respect to Oas1a as compared to Oas1 $\mathrm{g}$ is likely due to decreased usage of the unproductive splice site to begin with, but also note that the small differences observed in Oas1a expression levels could play a role in the aforementioned antiviral and apoptosis effects.

\section{Human Oas1 is regulated at the posttranscriptional level through productive and unproductive alternative splicing}

Human Oas1 differs quite significantly from the mouse Oas 1 paralogs, a finding that is perhaps not surprising given the volatile evolutionary history of the gene (Kumar et al. 2000; Hancks et al. 2015; Fish and Boissinot 2016). Despite differences between human Oas1 and mouse Oas1g, human Oas1 is extensively regulated at the posttranscriptional level through both productive and unproductive AS. Productive AS at the $3^{\prime}$ end of human Oas 1 gives rise to six isoforms (p42, p44(a/b), p46, p48, and p52) (Fig. 3A; Productive AS Events (Kjær et al. 2014). Expression of these isoforms varies in humans. For example, RNA-sequencing of IFN- $\alpha$ stimulated peripheral blood mononuclear cells (PBMCs) from two different healthy donors reveals one donor expresses primarily the $\mathrm{p} 44 \mathrm{a}$ isoform, while the other expresses primarily the p46 isoform (Fig. 3B). Genetic variation at the p46 spliceacceptor locus plays a role in this alteration of isoform abundance. There exists a single G/A SNP (rs10774671) in the p46 exon 6 splice-acceptor. Those with the $G$ allele predominantly produce $\mathrm{p} 46$, while the A allele leads to production of the other isoforms (Fig. 3A). Of the isoforms, the p46 isoform has been shown to have the greatest oligoadenylate synthetase activity, an effect mediated at least in part by defects in protein accumulation of the other isoforms (Bonnevie-Nielsen et al. 2005; Liu et al. 2017; Carey et al. 2019). As might be expected, production of the high activity isoform has been shown to dampen susceptibility to and/or severity of a variety of viral infections, including those mediated by West Nile virus (Lim et al. 2009), Epstein-Barr virus (Liu et al. 2017) and hepatitis $C$ virus (Knapp et al. 2003). However, there also appear to be costs associated with high OAS1 activity in humans, most notably in response to dengue virus-2 infection (Simon-Loriere et al. 2015). The $\mathrm{G}$ allele, which leads to production of the high activity p46 Oas 1 isoform, is associated with increased susceptibility to plasma leakage and shock in infected individuals, indicating immune overreaction could be triggered by increased OAS1 activity arising from altered AS (Simon-Loriere et al. 2015).

In addition to the productive splicing events at the $3^{\prime}$ end of the Oas1 transcript that lead to the generation of multiple isoforms, we identified another unproductive splicing event at the third splice junction (Fig. 3A; Unproductive AS Event). This unproductive splicing event is mediated by a $3^{\prime}$ splice site located $47 \mathrm{bp}$ into exon 3. When this unproductive splice site is selected, the frame of the transcript is shifted and a PTC is incorporated into the transcript, making it an ideal NMD substrate. This unproductive splice site is used frequently in a variety of cell types in response to a number of stimulation conditions (Fig. 3C-E). In general, $10 \%$ of spliced reads at this junction splice to the unproductive $3^{\prime}$ splice site, though it is worth noting all of the human samples were derived from whole-cell RNA and thus, might underestimate the frequency of usage of this unproductive splice site due to efficient degradation of PTC containing transcripts by the NMD decay machinery in the cytoplasm. In conclusion, though human Oas 1 and mouse Oas $1 \mathrm{~g}$ differ significantly, they are both regulated posttranscriptionally via AS.

\section{AS-NMD events are common in transcripts related to innate immunity}

While Oas1g contained one of the most frequently used AS-NMD events, it was not the only AS-NMD event found in genes related to the innate immune response. For example, in nuclear fractionation RNA-sequencing data from 
A
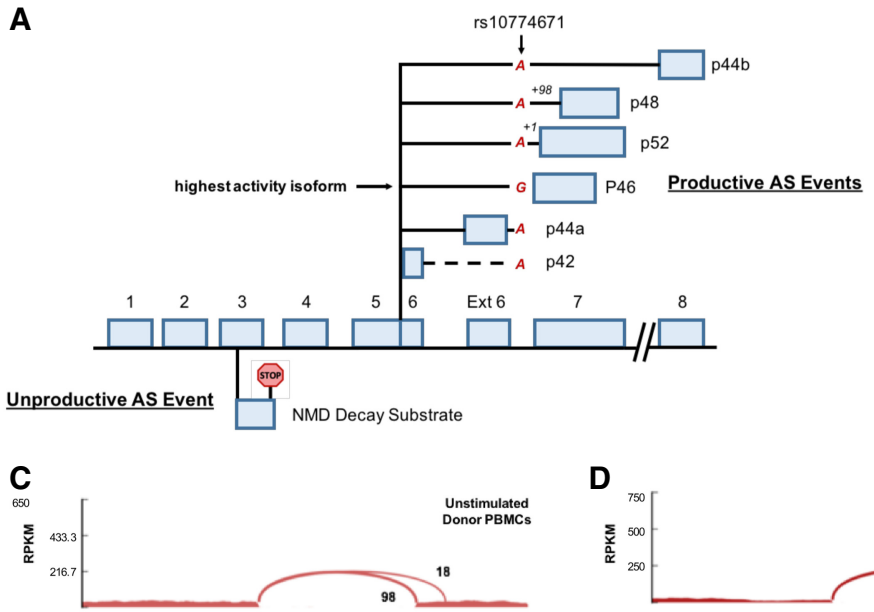

D
B 578

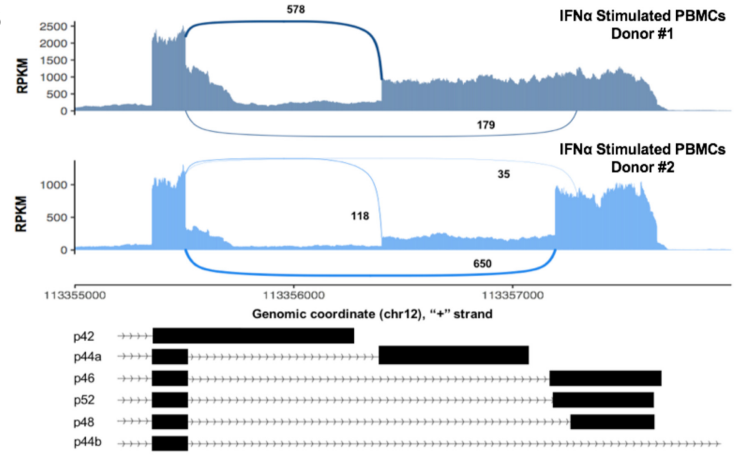

E
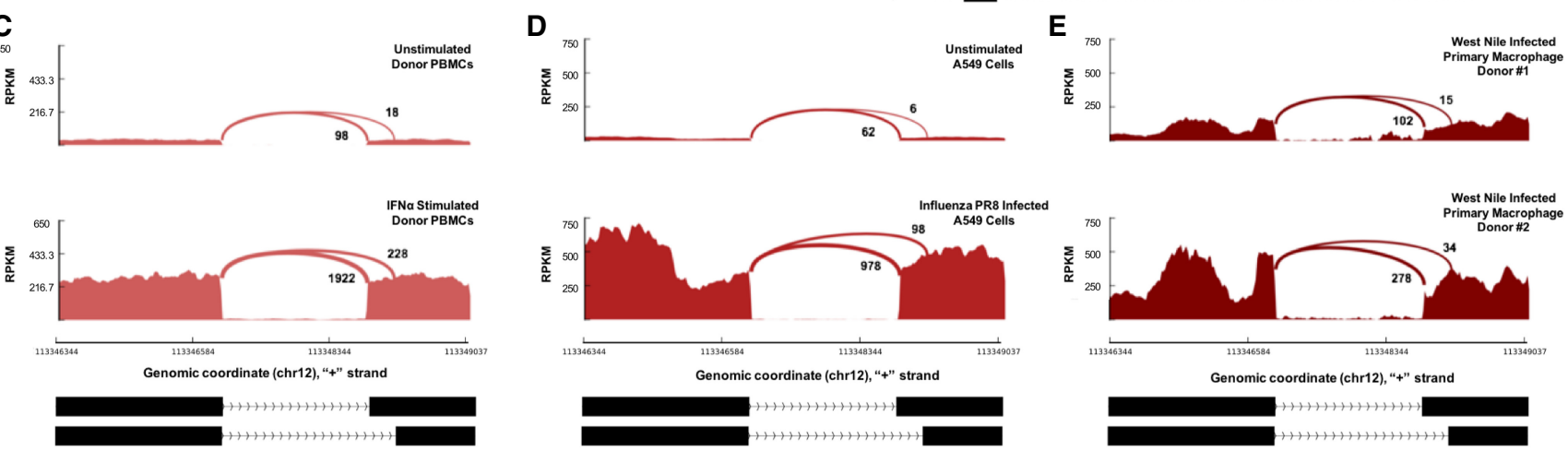

FIGURE 3. Human Oas1 is regulated at the posttranscriptional level through productive and unproductive AS. (A) Depiction of the mRNA splice isoforms found in human Oas1. There exists a single G/A SNP in the OAS1 exon 6 splice-acceptor (rs10774671), with the G variant producing the more active p46 isoform. (B) Sashimi plots depicting the productive AS event at the $3^{\prime}$ end of human Oas1. RNA is from PBMCs stimulated with IFN- $\alpha$ derived from two healthy donors. Genomic coordinates represent the hg19 genome assembly. (C-E) Sashimi plots for an AS-NMD event identified in exon 3 of human Oas1 from healthy donor derived PBMCs either unstimulated (top) or stimulated with IFN- $\alpha$ (C), A549 cells uninfected (top) or infected with influenza virus PR8 (D), or primary macrophages from two separate donors infected with West Nile virus (E). Genomic coordinates represent the hg19 genome assembly. All sequencing samples in Figure 3 are derived from whole-cell RNA.

mouse BMDMs stimulated with poly(l:C), we found significantly utilized cassette exon events that lead to a frameshift and incorporation of a PTC in the important innate immune response transcripts $M x 1, I K K \varepsilon$, and Oas/2 (Fig. $4 \mathrm{~A}-\mathrm{C})$. In each case, the exclusion of the cassette exon leads to an ideal NMD substrate. These events were also confirmed in the RAW264.7 macrophage cell line with RT-PCR with RNA from whole-cell fractions that had been stimulated with poly(l:C) for 4, 8, and $12 \mathrm{~h}$ (Fig. 4D-F).

To classify AS-NMD events globally, we utilized the tool SplAdder to predict and quantify AS events supported by an input sample (Kahles et al. 2016). A stringent confidence criterion was required to avoid including AS events derived from splicing noise. Then, only events that lead to frameshifts and/or PTC inclusion were selected. Among the list of AS-NMD events, as compared to a background of expressed genes, we observed significant enrichment for Gene Ontology (GO) terms associated with the innate immune response (Fig. 4G). With respect to the viral pathogen response, which is tasked with limiting viral replication through degradation of viral (as well as nonviral) mRNA and establishment of a cellular antiviral state, a host of factors involved with the response contain AS-
NMD events that are identified here or in other published work (Fig. 4H; Frankiw et al. 2019).

\section{DISCUSSION}

The robust and coordinated gene expression program involved in the defense against pathogens requires tight regulation. In this study, we sought to shed light on the role of AS-NMD in this regulation. We identified a frequently used unproductive splicing event in Oas1g, an important murine antiviral response factor, and show that forced productive splicing leads to increased Oas $1 \mathrm{~g}$ expression and further, an increased ability to clear virus. Additionally, we identified a number of other examples of unproductive splicing events in the innate immune response which could subject the corresponding transcript to decay via the NMD pathway.

With respect to Oas1g, what benefit might this AS event offer? The alternative splice site mediating this AS-NMD event is of comparable strength to the consensus $5^{\prime}$ splice site (Supplemental Fig. S1C). If possession of the greatest pathogen defense were the only goal of an organism, it seems unlikely this splice site would be retained. However, while pathogen defense systems can provide a 
A

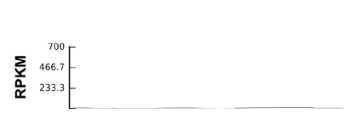

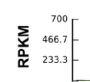
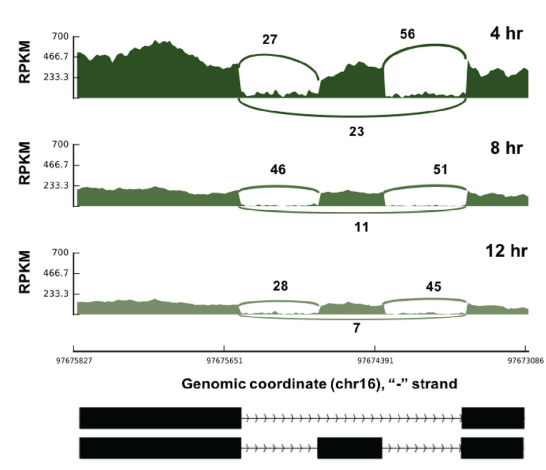

D

MX1

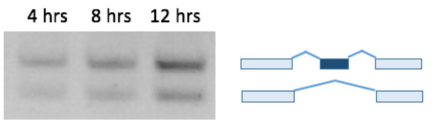

G

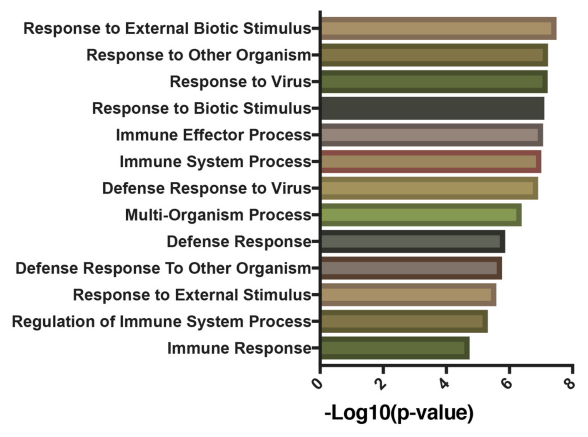

B
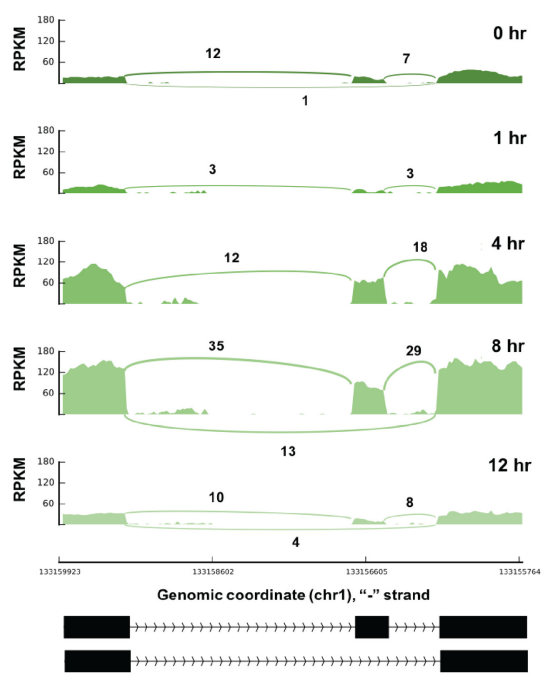

E

\section{政}

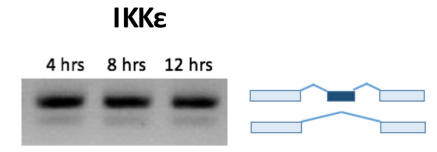

C
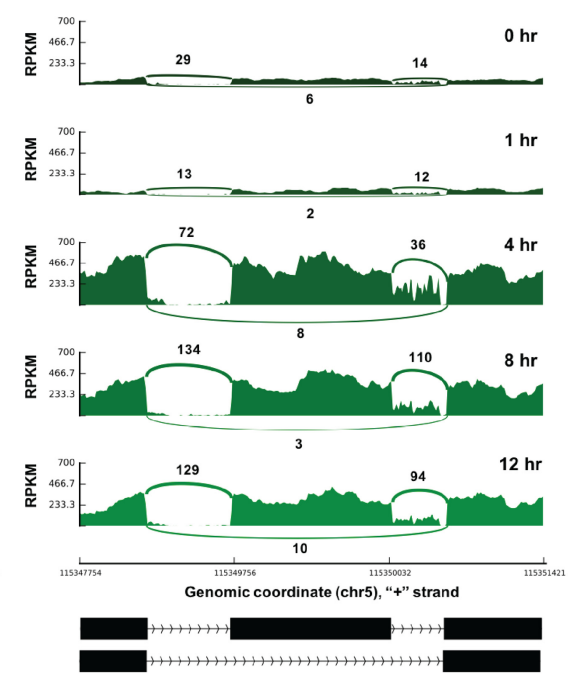

$\mathbf{F}$

OASL2

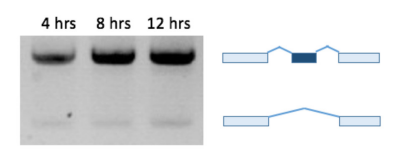

H

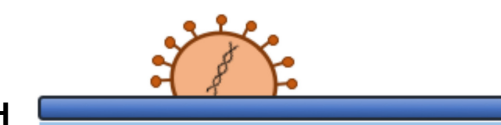

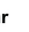

\section{(1)}

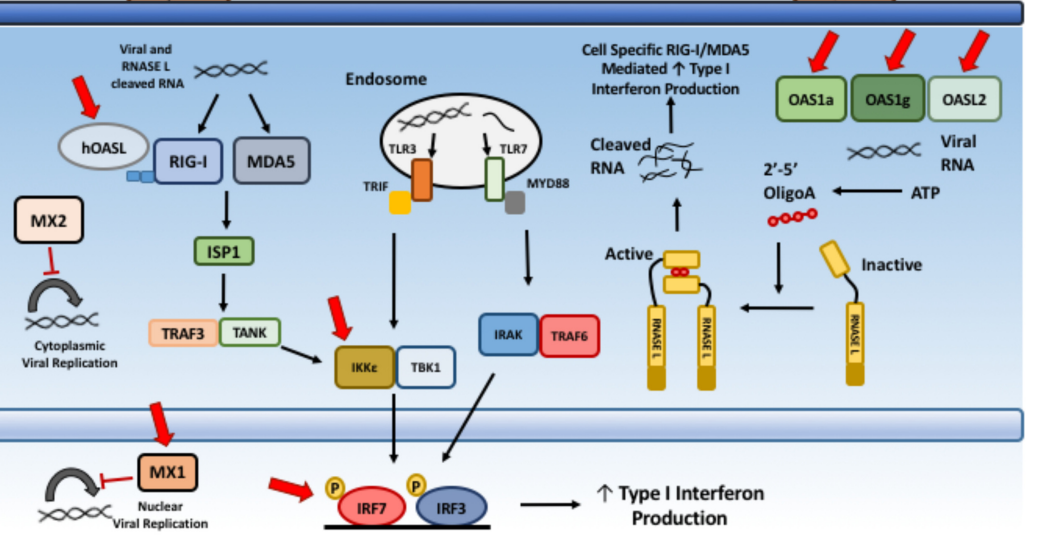

FIGURE 4. AS-NMD events are common in transcripts related to innate immunity. (A) Sashimi plots for an AS-NMD event identified in Mx1 from BMDMs stimulated with poly(l:C) for $0,1,4,8$, and $12 \mathrm{~h}$. Sequenced RNA was derived from the total nuclear fraction. The $y$-axis represents reads per kilobase of transcript, per million mapped reads (RPKM). (B) Same as A for IKKE. (C) Same as A for Oas/2. (D) RT-PCR of Mx1 upon stimulation with poly(l:C) for 4, 8, and $12 \mathrm{~h}$. (E) Same as D for IKKE. (F) Same as D for Oas/2. (G) GO terms enriched for AS-NMD events, as compared to a background of expressed genes. $(H)$ Schematic representation of major pathways in the viral pathogen response. Red arrows are shown above factors containing AS-NMD events. Data are representative of two independent experiments $(D-F)$. Genomic coordinates represent the mm9 genome assembly.

protective benefit, they also can cause collateral damage to a host. With respect to Oas1, its pathogen defense effects are repeatedly forfeited by a host due to the fact that its activity can be so detrimental (Kjaer et al. 2009; Carey et al. 2019). This is exemplified by the surprisingly high frequency of loss-of-function mutations in Oas 1 in primates (Carey et al. 2019), and the fact that OAS1 activity has been completely lost in several animal lineages, in- cluding teleost fish and insects (Kjaer et al. 2009). Moreover, while mice deficient for RNase $L$, the downstream effector of Oas1 in the 2-5A system, exhibit susceptibility to viral infection (Zhou et al. 1997), in the absence of infection they display significantly increased longevity (Andersen et al. 2007). Given the fact that host RNAs have been shown to be able to activate OAS enzymes, it is reasonable to hypothesize that the longevity 
effect is mediated, at least in part, by chronic 2-5A production (Molinaro et al. 2006; Dan et al. 2012; Li et al. 2017; Carey et al. 2019). With respect to the AS-NMD event we observed in Oas1g, we found removal of the unproductive splice site significantly increased the number of cells undergoing apoptosis. From this, it stands to reason that removal of the unproductive splice site, while improving the ability to limit viral infection, could negatively impact host fitness. In turn, we believe this splice site represents a compromise between the pathogen protective benefits and collateral damage associated with OAS1g activity.

A second question has to do with the manner with which this mitigation occurs. Innately, regulation at the posttranscriptional level through AS-NMD appears inefficient. Why spend the resources to transcribe a transcript if it is destined only for degradation? For one, the very fact that introns exist and are transient in nature argues against the idea that the cost of transcription is prohibitive (Lareau et al. 2007a). A significant majority of transcribed sequence ( $90 \%$ in humans) is spliced and discarded. Additionally, it is well understood that transcriptional regulation is largely a cooperative venture (Spitz and Furlong 2012), epitomized by complexes like the IFN- $\beta$ enhanceosome (Thanos and Maniatis 1995). As transcriptional regulation is not simply one protein interacting with one DNA sequence, but instead a multitude of proteins interacting with a host of other proteins and a variety of DNA sequences, it is possible that once transcriptional control has been placed on a system, changing it quantitatively is difficult as it requires the coordination of many different mutational events. Thus, secondary mechanisms must be brought into play to finetune the gene expression levels of particular transcripts. As such, we argue that the fine-tuning capabilities inherent to splicing-based posttranscriptional regulation far outweigh the cellular cost of additional transcription, especially in the context of a tightly regulated gene expression program like inflammation.

It remains unknown whether the AS-NMD event in Oas1 $g$ is regulated by an external input or whether a constant fraction of transcripts is discarded. The fact that the alternative splice site for Oas1g and Oas1a are identical in sequence and further, both junctions are nearly identical, supports the idea that trans-acting proteins might affect the process. Further, we notice a trend whereby increases in stimulation time accompany decreases in usage of the unproductive splice site (Fig. 1C), in essence arguing that this AS-NMD event acts as a break on Oas1g induction. It is possible that this trend is controlled by trans-acting proteins which promote the selection of the productive splice site over the unproductive splice site, and are induced by poly(I:C) and/or viral stimulation. Newly developed methods like RAP-MS (McHugh et al. 2015) and ChIRP-MS (Chu et al. 2015), which identify RNA binding proteins bound to RNAs of interest, could help discover in- teractions that have the ability to affect splice site selection.

In summary, we found a frequently used AS-NMD event in Oas1g. When the splice site that mediates this event is removed, we observed increased expression of Oas1g, an improved antiviral response, as well as an increase in the fraction of apoptotic cells. We hypothesize this event exists as a compromise between the pathogen protective benefits and collateral damage associated with OAS1 $\mathrm{g}$ activity. Similar unproductive splicing events were found throughout the innate immune response. While future studies should seek to understand the functional significance of individual events, this form of unproductive splicing has the potential to play a role in the regulation of gene expression in innate immunity.

\section{MATERIALS AND METHODS}

\section{Contact for reagent and resource sharing}

Further information and requests for resources and reagents should be directed to and will be fulfilled by the Lead Contact, David Baltimore (baltimo@caltech.edu).

\section{Experimental model and subject detail}

\section{Cell culture}

All cell lines were maintained at $37^{\circ} \mathrm{C}$. Human embryonic kidney cells (HEK293T) from ATCC were cultured in DMEM supplemented with 10\% FBS and 1\% Pen/Strep. RAW 264.7 murine macrophages from ATCC were cultured in DMEM supplemented with $10 \% \mathrm{FBS}$ and $1 \%$ Pen/Strep. Cell lines were maintained at $37^{\circ} \mathrm{C}$ in $5 \% \mathrm{CO}_{2}$.

\section{Method detail}

\section{RNA isolation}

Total RNA was purified from BMDMs using TRlzol reagent (Ambion) as per the manufacturer's instructions. Genomic DNA in RNA purifications was eliminated through treatment with Turbo DNase (Thermo Fisher Scientific) for $30 \mathrm{~min}$ at $37^{\circ} \mathrm{C}$.

\section{RT-PCR of splice isoforms}

Total RNA was isolated using Tri reagent solution and digested with DNase I (Invitrogen). RT reactions were performed in $20 \mu \mathrm{L}$ (20 mM DTT, 2× ProtoScript II Reverse Transcriptase Reaction Buffer [NEB], 1 mM dNTPs, 40 U Murine RNase Inhibitor [NEB], and 200 U ProtoScript II [NEB] Reverse Transcriptase) with 500$1000 \mathrm{ng}$ RNA. Reaction incubated in thermocycler with the following program: $1.42^{\circ} \mathrm{C}$ for $60 \mathrm{~min}, 2.65^{\circ} \mathrm{C}$ for $20 \mathrm{~min}$. PCR was performed using 05 Hot Start High-Fidelity DNA Polymerase (NEB) and gene-specific primers. 


\section{CRISPR experiments}

RAW 264.7 cell lines were grown in individual $10 \mathrm{~cm}$ plates. Twenty thousand cells were plated in a cell well plate and left overnight to adhere. Following adherence, lentivirus expressing Cas9 and either the Oas1a/g guide or a scramble control guide was added to the cells. Forty-eight hours later, infected cells were selected with puromycin, which was added at a concentration of $3.75 \mu \mathrm{g} / \mathrm{mL}$. Following $72 \mathrm{~h}$ of selection, cells infected with the Oas1a/g guide were single-cell plated in 96-well plates. Clones were passed to six-well plates following $5 \mathrm{~d}$ of growth, at which point genotyping was performed. Cells infected with the scramble control guide were passaged as a bulk infected population, with independent biological replicates representing cells independently infected and puromycin selected.

\section{Poly(l:C) stimulations and gene expression quantification experiments}

RAW 264.7 cell lines were grown in individual $10 \mathrm{~cm}$ plates. Twelve hours prior to infection, cells were counted and plated at a density of 350,000 cells/well in six-well plates. Following adherence, $5 \mu \mathrm{g} / \mathrm{mL}$ of Poly(l:C) (Sigma) was added. Following the stimulation, total RNA was isolated using Tri reagent solution and digested with DNase I (Invitrogen). RT reactions were performed in $20 \mu \mathrm{L}(20 \mathrm{mM}$ DTT, 2x ProtoScript II Reverse Transcriptase Reaction Buffer [NEB], 1 mM dNTPs, 40 U Murine RNase Inhibitor [NEB], and 200 U ProtoScript II [NEB] Reverse Transcriptase) with 500-1000 ng RNA. Reaction incubated in thermocycler with the following program: $1.42^{\circ} \mathrm{C}$ for $60 \mathrm{~min}, 2.65^{\circ} \mathrm{C}$ for $20 \mathrm{~min}$. Cellular RNA was quantified using TaqMan PCR probes (see Supplemental Table 1 for probes; Thermo Fisher).

\section{EMCV infection experiment}

RAW 264.7 cell lines were grown in individual $10 \mathrm{~cm}$ plates. Twelve hours prior to infection, cells were counted and plated at a density of 350,000 cells/well in six-well plates. Following adherence, EMCV was added at the specified MOI for the specified amount of time. Following the infection, cells were lysed in TRIzol. Viral RNA was quantified using SYBR green (Kapa Biosystems). Cellular Oas1g and Oas1a RNA was quantified using TaqMan PCR probes (Thermo Fisher). All oligonucleotide sequences can be found in Supplemental Table 1.

\section{Annexin $V$ experiment}

RAW 264.7 cell lines were grown in individual $10 \mathrm{~cm}$ plates. Twelve hours prior to infection, cells were counted and plated at a density of 125,000 cells/well in 12-well plates. Twenty-four hours after plating, cells were stained with Annexin V APC Ready Flow Conjugate (Thermo Fisher). Samples were analyzed on a MACSQuant10 Flow Cytometry machine (Miltenyi). Data was analyzed with FlowJo 10.2.

\section{Data download}

Raw RNA-sequencing samples in FASTQ format were downloaded from the Gene Expression Omnibus (GEO) database. Mouse bone-marrow macrophages derived data can be found under accession number GSE122543 (SRR8187909, SRR8187910, SRR8187911, SRR8187912, SRR8187913). Human PBMC data can be found under accession number GSE72502 (SRR2192716, SRR2192719, SRR2192720). Human A549 data can be found under accession number GSE121155 (SRR8037285, SRR8037289). Human primary macrophage data can be found under accession number GSE40718 (SRR566252, SRR566260).

\section{RNA-sequencing analysis}

Sequencing was performed on a HiSeq 2500 High-Throughput Sequencer (Illumina). All previously downloaded RNA-seq samples were aligned using a pipeline based on the STAR aligner (version 2.6.0a) (Dobin et al. 2013). The STAR software (version 2.6.0a) was used in a two-pass mode. The first pass identifies nonannotated junctions in the input, allowing for the construction of a genome index containing nonannotated junctions. The second pass alignment is then performed against the junction-aware index. Following alignment, Portcullis (Mapleson et al. 2018) was used to filter invalid splice junctions from the aligned BAM file. Isoform expression was quantified using the raw fastq files and the mouse reference transcriptome $\mathrm{mm} 9$ as input for Kallisto (v.0.45.0) (Bray et al. 2016). The resultant normalized transcript frequencies were provided to the R package Sleuth for differential analysis (v.0.30.0) (Pimentel et al. 2017). Alternative splicing events were detected and quantified on produced BAM files using the SplAdder toolkit (Kahles et al. 2016) as per Kahles et al. (2018). Only events that passed the highest confidence level parameter were selected. Unproductive splicing events were determined using a custom Python script. Alternative splicing sashimi plots across an entire gene were generated with ggsashimi (Garrido-Martín et al. 2018) whereby only junctions with greater than five supporting RNA-seq reads were plotted, while AS sashimi plots centered at an individual junction were generated with MISO (Katz et al. 2010). $\Psi$ estimates, as well as confidence intervals over estimates shown in histograms of Figure $1 \mathrm{C}$ were generated using only reads from the most abundant read length.

\section{Alternative junction usage analysis}

Isoform expression was quantified using the raw fastq files and the mouse reference transcriptome $\mathrm{mm} 9$ as input for Kallisto (v.0.45.0). Only isoforms with a TPM greater than 10 were considered. Junctions from the most abundant isoform for each gene were selected, as long as there were eight supporting reads for the junction. Alternative junction usage was calculated by comparing the number of reads that overlap a given selected junction, but did not utilize the same $5^{\prime}$ and/or $3^{\prime}$ splice site, to the total number of reads at a junction.

\section{Quantification and statistical analysis}

All statistical analysis was performed in Python (version 2.7.9). Unless otherwise indicated in figure legends, statistical significance measurements were marked as follows: $\left(^{*}\right)$ denotes $P<$

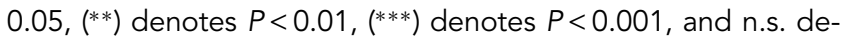
notes not significant. 


\section{SUPPLEMENTAL MATERIAL}

Supplemental material is available for this article.

\section{ACKNOWLEDGMENTS}

The authors would like to thank Bert Semler (Department of Microbiology and Molecular Genetics, University of California, Irvine) for providing EMCV. The authors would also like to thank Megan Bergkessel (Department of Biology and Bioengineering, California Institute of Technology) and Prashant Bhat (Department of Biology and Bioengineering, California Institute of Technology) for experimental advice. This work was funded by an endowment provided by the Raymond and Beverly Sackler Foundation.

Author contributions: L.F. and D.B. conceived and designed experiments with input from M.M., G.L., and A.J. L.F. conducted experiments and analyzed data. The manuscript was written by L.F. and D.B.

Received October 29, 2019; accepted November 13, 2019.

\section{REFERENCES}

Andersen JB, Li XL, Judge CS, Zhou A, Jha BK, Shelby S, Zhou L, Silverman RH, Hassel BA. 2007. Role of 2-5A-dependent RNase$L$ in senescence and longevity. Oncogene 26: 3081. doi:10 .1038/sj.onc.1210111

Bitton DA, Atkinson SR, Rallis C, Smith GC, Ellis DA, Chen YY, Malecki M, Codlin S, Lemay J-F, Cotobal C, et al. 2015. Widespread exon skipping triggers degradation by nuclear RNA surveillance in fission yeast. Genome Res 25: 884-896. doi:10.1101/gr .185371 .114

Bonnevie-Nielsen V, Leigh Field L, Lu S, Zheng D-J, Li M, Martensen PM, Nielsen TB, Beck-Nielsen H, Lau Y-L, Pociot F. 2005. Variation in antiviral $2^{\prime}, 5^{\prime}$-oligoadenylate synthetase $\left(2^{\prime} 5^{\prime} \mathrm{AS}\right)$ enzyme activity is controlled by a single-nucleotide polymorphism at a splice-acceptor site in the OAS1 gene. Am J Hum Genet 76: 623-633. doi:10.1086/429391

Bray NL, Pimentel H, Melsted P, Pachter L. 2016. Near-optimal probabilistic RNA-seq quantification. Nat Biotechnol 34: 525. doi:10 $.1038 / n b t .3519$

Carey CM, Govande AA, Cooper JM, Hartley MK, Kranzusch PJ, Elde NC. 2019. Recurrent loss-of-function mutations reveal costs to OAS1 antiviral activity in primates. Cell Host Microbe 25: 336-343. doi:10.1016/j.chom.2019.01.001

Carocci M, Bakkali-Kassimi L. 2012. The encephalomyocarditis virus. Virulence 3: 351-367. doi:10.4161/viru.20573

Castelli JC, Hassel BA, Wood KA, Li X-L, Amemiya K, Dalakas MC, Torrence PF, Youle RJ. 1997. A study of the interferon antiviral mechanism: apoptosis activation by the 2-5A system. J Exp Med 186: 967-972. doi:10.1084/jem.186.6.967

Chen J, Chen ZJ. 2013. Regulation of NF-kB by ubiquitination. Curr Opin Immunol 25: 4-12. doi:10.1016/j.coi.2012.12.005

Chu C, Zhang QC, Da Rocha ST, Flynn RA, Bharadwaj M, Calabrese JM, Magnuson T, Heard E, Chang HY. 2015. Systematic discovery of Xist RNA binding proteins. Cell 161: 404-416. doi:10 $.1016 / j$.cell.2015.03.025

Dan M, Zheng D, Field LL, Bonnevie-Nielsen V. 2012. Induction and activation of antiviral enzyme 2', 5'-oligoadenylate synthetase by in vitro transcribed insulin mRNA and other cellular RNAs. Mol Biol Rep 39: 7813-7822. doi:10.1007/s11033-012-1624-x

Dobin A, Davis CA, Schlesinger F, Drenkow J, Zaleski C, Jha S, Batut P, Chaisson M, Gingeras TR. 2013. STAR: ultrafast universal RNA-seq aligner. Bioinformatics 29: 15-21. doi:10.1093/bioinformatics/ bts635

Elkhateeb E, Tag-El-Din-Hassan HT, Sasaki N, Torigoe D, Morimatsu M, Agui T. 2016. The role of mouse 2', 5'-oligoadenylate synthetase 1 paralogs. Infect Genet Evol 45: 393-401. doi:10 .1016/j.meegid.2016.09.018

Fish I, Boissinot S. 2016. Functional evolution of the OAS1 viral sensor: insights from old world primates. Infect Genet Evol 44: 341-350. doi:10.1016/j.meegid.2016.07.005

Frankiw L, Majumdar D, Burns C, Vlach L, Moradian A, Sweredoski MJ, Baltimore D. 2019. Bud13 promotes a type I interferon response by countering intron retention in Irf7. Mol Cell 73: 803-814. doi:10.1016/j.molcel.2018.11.038

Garrido-Martín D, Palumbo E, Guigó R, Breschi A. 2018. ggsashimi: Sashimi plot revised for browser- and annotation-independent splicing visualization. PLoS Comput Biol 14: e1006360. doi:10 .1371/journal.pcbi.1006360

Gautier EL, Shay T, Miller J, Greter M, Jakubzick C, Ivanov S, Helft J, Chow A, Elpek KG, Gordonov S, et al. 2012. Gene-expression profiles and transcriptional regulatory pathways that underlie the identity and diversity of mouse tissue macrophages. Nat Immunol 13: 1118. doi:10.1038/ni.2419

Hancks DC, Hartley MK, Hagan C, Clark NL, Elde NC. 2015. Overlapping patterns of rapid evolution in the nucleic acid sensors cGAS and OAS1 suggest a common mechanism of pathogen antagonism and escape. PLoS Genet 11: e1005203. doi:10.1371/ journal.pgen.1005203

Hao S, Baltimore D. 2009. The stability of mRNA influences the temporal order of the induction of genes encoding inflammatory molecules. Nat Immunol 10: 281. doi:10.1038/ni.1699

Hearl WG, Johnston MI. 1987. Accumulation of 2',5'-oligoadenylates in encephalomyocarditis virus-infected mice. J Virol 61: 15861592.

Jangi M, Sharp PA. 2014. Building robust transcriptomes with master splicing factors. Cell 159: 487-498. doi:10.1016/j.cell.2014.09 .054

Kahles A, Ong CS, Zhong Y, Rätsch G. 2016. SplAdder: identification, quantification and testing of alternative splicing events from RNASeq data. Bioinformatics 32: 1840-1847. doi:10.1093/bioinfor matics/btw076

Kahles A, Lehmann K-V, Toussaint NC, Hüser M, Stark SG, Sachsenberg T, Stegle O, Kohlbacher O, Sander C, CaesarJohnson SJ, et al. 2018. Comprehensive analysis of alternative splicing across tumors from 8,705 patients. Cancer Cell 34: 211 224. doi:10.1016/j.ccell.2018.07.001

Kakuta S, Shibata S, Iwakura Y. 2002. Genomic structure of the mouse 2', 5'-oligoadenylate synthetase gene family. J Interferon Cytokine Res 22: 981-993. doi:10.1089/10799900260286696

Katz Y, Wang ET, Airoldi EM, Burge CB. 2010. Analysis and design of RNA sequencing experiments for identifying isoform regulation. Nat Methods 7: 1009-1015. doi:10.1038/nmeth.1528

Kervestin S, Jacobson A. 2012. NMD: a multifaceted response to premature translational termination. Nat Rev Mol Cell Biol 13: 700. doi:10.1038/nrm3454

Kjaer KH, Poulsen JB, Reintamm T, Saby E, Martensen PM, Kelve M, Justesen J. 2009. Evolution of the 2'-5'-oligoadenylate synthetase family in eukaryotes and bacteria. J Mol Evol 69: 612. doi:10.1007/ s00239-009-9299-1

Kjær K, Pahus J, Hansen M, Poulsen J, Christensen E, Justesen J, Martensen P. 2014. Mitochondrial localization of the OAS1 p46 isoform associated with a common single nucleotide polymorphism. BMC Cell Biol 15: 33. doi:10.1186/1471-2121-15-33

Knapp S, Yee LJ, Frodsham AJ, Hennig BJW, Hellier S, Zhang L, Wright M, Chiaramonte M, Graves M, Thomas HC, et al. 2003. Polymorphisms in interferon-induced genes and the outcome of 
hepatitis $C$ virus infection: roles of MxA, OAS-1 and PKR. Genes Immun 4: 411-419. doi:10.1038/sj.gene.6363984

Kontoyiannis D, Pasparakis M, Pizarro TT, Cominelli F, Kollias G. 1999. Impaired on/off regulation of TNF biosynthesis in mice lacking TNF AU-rich elements: implications for joint and gut-associated immunopathologies. Immunity 10: 387-398. doi:10.1016/S10747613(00)80038-2

Kumar S, Mitnik C, Valente G, Floyd-Smith G. 2000. Expansion and molecular evolution of the interferon-induced $2^{\prime}-5^{\prime}$ oligoadenylate synthetase gene family. Mol Biol Evol 17: 738-750. doi:10 .1093/oxfordjournals.molbev.a026352

Lareau LF, Brooks AN, Soergel DA, Meng Q, Brenner SE. 2007a. The coupling of alternative splicing and nonsense-mediated mRNA decay. Adv Exp Med Biol 623: 190-211. doi:10.1007/978-0387-77374-2_12

Lareau LF, Inada M, Green RE, Wengrod JC, Brenner SE. 2007b. Unproductive splicing of SR genes associated with highly conserved and ultraconserved DNA elements. Nature 446: 926. doi:10.1038/nature05676

Leppek K, Schott J, Reitter S, Poetz F, Hammond MC, Stoecklin G. 2013. Roquin promotes constitutive mRNA decay via a conserved class of stem-loop recognition motifs. Cell 153: 869-881. doi:10 .1016/j.cell.2013.04.016

Lewis BP, Green RE, Brenner SE. 2003. Evidence for the widespread coupling of alternative splicing and nonsense-mediated mRNA decay in humans. Proc Natl Acad Sci 100: 189-192. doi:10 $.1073 /$ pnas. 0136770100

Li Y, Banerjee S, Goldstein SA, Dong B, Gaughan C, Rath S, Donovan J, Korennykh A, Silverman RH, Weiss SR. 2017. Ribonuclease $L$ mediates the cell-lethal phenotype of doublestranded RNA editing enzyme ADAR1 deficiency in a human cell line. Elife 6: e25687. doi:10.7554/eLife.25687

Lim JK, Lisco A, McDermott DH, Huynh L, Ward JM, Johnson B, Johnson H, Pape J, Foster GA, Krysztof D, et al. 2009. Genetic variation in OAS1 is a risk factor for initial infection with West Nile virus in man. PLoS Pathog 5: e1000321. doi:10.1371/journal.ppat .1000321

Liu X, Xing H, Gao W, Yu D, Zhao Y, Shi X, Zhang K, Li P, Yu J, Xu W, et al. 2017. A functional variant in the OAS1 gene is associated with Sjögren's syndrome complicated with HBV infection. Sci Rep 7: 17571. doi:10.1038/s41598-017-17931-9

Mapleson D, Venturini L, Kaithakottil G, Swarbreck D. 2018. Efficient and accurate detection of splice junctions from RNA-seq with Portcullis. Gigascience 7: giy131. doi:10.1093/gigascience/giy131

McGlincy NJ, Smith CW. 2008. Alternative splicing resulting in nonsense-mediated mRNA decay: what is the meaning of nonsense? Trends Biochem Sci 33: 385-393. doi:10.1016/j.tibs.2008.06.001

McHugh CA, Chen C-K, Chow A, Surka CF, Tran C, McDonel P, Pandya-Jones A, Blanco $M$, Burghard $C$, Moradian A, et al. 2015. The Xist IncRNA interacts directly with SHARP to silence transcription through HDAC3. Nature 521: 232. doi:10.1038/ nature 14443

Mendell JT, Sharifi NA, Meyers JL, Martinez-Murillo F, Dietz HC. 2004. Nonsense surveillance regulates expression of diverse classes of mammalian transcripts and mutes genomic noise. Nat Genet 36: 1073. doi:10.1038/ng1429
Molinaro RJ, Jha BK, Malathi K, Varambally S, Chinnaiyan AM, Silverman RH. 2006. Selection and cloning of poly ( $\mathrm{rC}$ )-binding protein 2 and Raf kinase inhibitor protein RNA activators of $2^{\prime}$, 5 '-oligoadenylate synthetase from prostate cancer cells. Nucleic Acids Res 34: 6684-6695. doi:10.1093/nar/gkl968

O'Connell RM, Rao DS, Baltimore D. 2012. microRNA regulation of inflammatory responses. Annu Rev Immunol 30: 295-312. doi:10 .1146/annurev-immunol-020711-075013

Pan Q, Shai O, Lee LJ, Frey BJ, Blencowe BJ. 2008. Deep surveying of alternative splicing complexity in the human transcriptome by high-throughput sequencing. Nat Genet 40: 1413. doi:10.1038/ ng.259

Perales-Linares R, Navas-Martin S. 2013. Toll-like receptor 3 in viral pathogenesis: friend or foe? Immunology 140: 153-167. doi:10 $.1111 /$ imm.12143

Pimentel H, Bray NL, Puente S, Melsted P, Pachter L. 2017. Differential analysis of RNA-seq incorporating quantification uncertainty. Nat Methods 14: 687. doi:10.1038/nmeth.4324

Silverman RH. 2007. Viral encounters with 2', 5'-oligoadenylate synthetase and RNase $\mathrm{L}$ during the interferon antiviral response. $J$ Virol 81: 12720-12729. doi:10.1128/JVI.01471-07

Simon-Loriere E, Lin R-J, Kalayanarooj SM, Chuansumrit A, Casademont I, Lin S-Y, Yu H-P, Lert-Itthiporn W, Chaiyaratana W, Tangthawornchaikul N, et al. 2015. High anti-dengue virus activity of the OAS gene family is associated with increased severity of dengue. J Infect Dis 212: 2011-2020. doi:10.1093/infdis/jiv321

Smale ST, Natoli G. 2014. Transcriptional control of inflammatory responses. Cold Spring Harb Perspect Biol 6: a016261. doi:10 $.1101 /$ cshperspect.a016261

Smale ST, Tarakhovsky A, Natoli G. 2014. Chromatin contributions to the regulation of innate immunity. Annu Rev Immunol 32: 489511. doi:10.1146/annurev-immunol-031210-101303

Spitz F, Furlong EE. 2012. Transcription factors: from enhancer binding to developmental control. Nat Rev Genet 13: 613. doi:10 $.1038 / \mathrm{nrg} 3207$

Thanos D, Maniatis T. 1995. Virus induction of human IFN $\beta$ gene expression requires the assembly of an enhanceosome. Cell 83: 1091-1100. doi:10.1016/0092-8674(95)90136-1

Wang ET, Sandberg R, Luo S, Khrebtukova I, Zhang L, Mayr C, Kingsmore SF, Schroth GP, Burge CB. 2008. Alternative isoform regulation in human tissue transcriptomes. Nature 456: 470. doi:10.1038/nature07509

Weischenfeldt J, Waage J, Tian G, Zhao J, Damgaard I, Jakobsen JS, Kristiansen K, Krogh A, Wang J, Porse BT. 2012. Mammalian tissues defective in nonsense-mediated mRNA decay display highly aberrant splicing patterns. Genome Biol 13: R35. doi:10.1186/gb2012-13-5-r35

Yeo G, Burge CB. 2004. Maximum entropy modeling of short sequence motifs with applications to RNA splicing signals. J Comput Biol 11: 377-394. doi:10.1089/1066527041410418

Zhou A, Paranjape J, Brown TL, Nie H, Naik S, Dong B, Chang A, Trapp B, Fairchild R, Colmenares C, et al. 1997. Interferon action and apoptosis are defective in mice devoid of $2^{\prime}, 5^{\prime}$-oligoadenylate-dependent RNase L. EMBO J 16: 6355-6363. doi:10.1093/ emboj/16.21.6355 

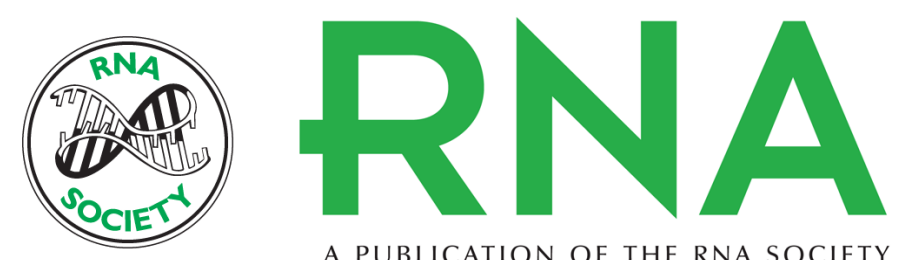

A PUBLICATION OF THE RNA SOCIETY

\section{Alternative splicing coupled with transcript degradation modulates OAS1g antiviral activity}

Luke Frankiw, Mati Mann, Guideng Li, et al.

RNA 2020 26: 126-136 originally published online November 18, 2019

Access the most recent version at doi:10.1261/rna.073825.119

\section{Supplemental http://rnajournal.cshlp.org/content/suppl/2019/11/18/rna.073825.119.DC1 Material}

References This article cites 56 articles, 7 of which can be accessed free at: http://rnajournal.cshlp.org/content/26/2/126.full.html\#ref-list-1

Creative This article is distributed exclusively by the RNA Society for the first 12 months after the Commons License full-issue publication date (see http://rnajournal.cshlp.org/site/misc/terms.xhtml). After 12 months, it is available under a Creative Commons License (Attribution-NonCommercial 4.0 International), as described at http://creativecommons.org/licenses/by-nc/4.0/.

Email Alerting
Service

Receive free email alerts when new articles cite this article - sign up in the box at the top right corner of the article or click here.

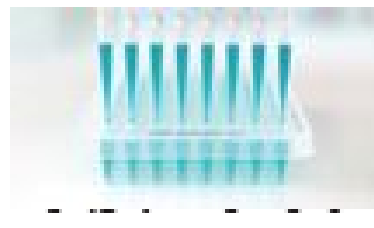

Providing Precise Solutions for your research.

To subscribe to $R N A$ go to:

http://rnajournal.cshlp.org/subscriptions

(C) 2020 Frankiw et al.; Published by Cold Spring Harbor Laboratory Press for the RNA Society 